\title{
Extending IM beyond the Reference \\ Desk: A Case Study on the Integration of Chat Reference and Library-Wide Instant Messaging Network
}

Ian Chan, Pearl Ly, and Yvonne Meulemans

\begin{abstract}
Openfire is an open-source instant messaging (IM) network and a single unified application that meets the needs of chat reference and internal communication. In Fall 2009, the California State University San Marcos (CSUSM) Library began using Openfire and other Jive software IM technologies to simultaneously improve our existing IM-integrated chat reference software and implement an internal IM network. This case study describes the chat reference and internal communications environment at the CSUSM Library and the selection, implementation, and evaluation of Openfire. In addition, the authors discuss the benefits of deploying an integrated IM and chat reference network.
\end{abstract}

\section{INTRODUCTION}

Instant messaging (IM) has become a prevalent contact point for library patrons to get information and reference help, commonly known as chat reference or virtual reference. However, IM can also offer a unique method of communication between library staff. Librarians are able to rapidly exchange information synchronously or asynchronously in an informal way. IM provides another means of building relationships within the library organization and can improve teamwork.

Many different chat-reference software packages are widely used by libraries, including QuestionPoint, Meebo, and LibraryH3lp. Less commonly used is Openfire

(www.igniterealtime.org/projects/openfire), an open-source IM network and a single unified application that uses the Extensible Messaging and Presence Protocol (XMPP), a widely adopted open protocol for IM.

Since 2009, the California State University San Marcos (CSUSM) Kellogg Library has used Openfire for chat reference and internal IM communication. Openfire was relatively easy to set up and administer by the Web Development Librarian. Librarians and library users have found the IM interface to be intuitive. In addition to helpful chat reference features such as statistics capture, queues, transfer, linking to Meebo widgets, Openfire offers the unique capability to host an internal IM network within the library.

Ian Chan (ichan@csusm.edu) is Web Development Librarian, California State University San Marcos, Pearl Ly (pmly@pasadena.edu) is Access Services \& Emerging Technologies Librarian, Pasadena Community College, Pasadena, and Yvonne Meulemans (ymeulema@csusm.edu) is Information Literacy Program Coordinator, California State University San Marcos, California. 
In this article, the authors present a literature review on IM as a workplace communication tool and its successful use in libraries for chat reference services. A case study on the selection, implementation, and evaluation of Openfire for use in chat reference and as an internal network will be discussed. In addition, survey results on the library staff use of the internal IM network and its implications for collaboration and increased communication are shared.

\section{LITERATURE REVIEW}

Although there is a great deal of literature on IM for library reference services, publications on the use of IM in libraries for internal communications do not appear in the professional literature. A review of library and information science (LIS) literature has revealed very limited work on this aspect of instant messaging. However, a wider literature review in the fields of communications, computer science, and business, indicates there is growing interest in studying the benefits of IM within organizations.

\section{Instant Messaging in the Workplace}

In the workplace, IM can offer a cost-effective means of connecting in real-time and may increase communication effectiveness between employees. It offers a number of advantages over email, telephone, and face-to-face that we will discuss further in the following section. Within the academic library, IM offers the possibility of not only improving access to librarians for research help but also provides the opportunity to enhance communication and collaboration throughout the entire organization.

Research findings indicate that IM allows coworkers to maintain a sense of connection and context that is different from email, face-to-face (FTF), and phone conversations. ${ }^{1}$ Each IM conversation is designed to display as a single textual thread with one window per conversation. The contributions from each person in the discussion are clearly indicated and it is easy to review what has been said. This design supports the intermittent reconnection of conversation and in contrast to email, "intermittent instant messages were thought to be more immersive and to give more of a sense of a shared space and context than such email exchanges."2 Through the use of IM, coworkers gain a highly interactive channel of communication that is not available via other methods of communication. ${ }^{3}$

Phone and FTF conversations are two of the most common forms of interruption within the workplace. ${ }^{4}$ However, Garrett and Danziger found that "instant messaging in the workplace simultaneously promotes more frequent communications and reduces interruptions."5

Participants reported they were better able to manage disruptions using IM and that IM did not increase their communication time. The findings of this study revealed that some communication that otherwise may have occurred over email, by telephone, or in-person were instead delivered via IM. This likely contributed to the reduced interruptions because IM does not require full and immediate attention unlike a phone call or face-to-face communication. In addition, IM study participants reported the ability to negotiate their availability through postponing conversations, 
and these findings support earlier studies suggesting IM is less intrusive than traditional communication methods for determining availability of coworkers. ${ }^{6}$

A number of research studies show that IM improves teamwork and is useful for discussing complex tasks. Huang, Hung, and Chen compared the effectiveness of email and IM and the number of new ideas; they found that groups utilizing IM generated more ideas than the email groups. ${ }^{7}$ They suggested that the spontaneous and rapid interchanges typical of IM facilitates brainstorming between team members. The information that is uniquely visible through IM and the ease of sending messages help create opportunities for spontaneous dialog. This is supported by a study by Quan-Haase, Cothrel, and Wellman, which found IM promotes team interaction by indicating the likelihood of a faster response. ${ }^{8}$ Ou et al. also suggest IM has "potential to empower teamwork by establishing social networks and facilitating knowledge sharing among organizational members." IM can enhance the social connectedness of coworkers through its focus on contact lists and instant, opportunistic interactivity.

The informal and personalized nature of IM allows workers to build relationships while promoting the sharing of information. Cho, Trier, and Kim suggest that the use of IM as a communication tool encourages unplanned virtual hallway discussions that may be difficult for those located in different parts of a building, campus, or in remote locations. ${ }^{10}$ IM can build relationships between teams and organizations where members are in physically separated locations. However, Cho, Trier, and Kim also note that IM is more successful in building relationships between coworkers who already have an existing relationship. Wu et al. argue that by helping to build the social network within the organization, instant messaging can contribute to increased productivity. ${ }^{11}$

Several studies have cautioned that IM, like other forms of communication, requires organizational guidelines on usage and best practices. Mahatanankoon suggests that productivity or job satisfaction may decrease without policies and workplace norms that guide IM use. ${ }^{12}$ Other research indicates that personality, employee status, and working style may affect the usefulness of IM for individual employees. ${ }^{13}$ Some workers may find the multitasking nature of IM to work in their favor while those who prefer sequential task completion may find IM disruptive. The hierarchy of work relationships and the nature of managerial styles are likely to have an impact on the use of IM as well.

While there are no research findings associated with the use of IM for internal communication within libraries, there are articles encouraging its use. Breeding writes of the potential for IM to bring about "a level of collaboration that only rarely occurs with the store-and-forward model of traditional e-mail." ${ }^{14}$ Fink provides a concise introduction to the advantages of using internal IM for communication between library staff. ${ }^{15}$ In addition, he provides an overview of the implementation and success of the Openfire-based IM network at McMaster University. 


\section{Success of Chat Reference in Libraries}

IM-based chat reference gives libraries the means to more easily offer low-cost delivery of synchronous, real-time research assistance to their users, commonly referred to as "chat reference." Although libraries have used IM for the last decade and many currently subscribe to QuestionPoint, a collaborative virtual reference service through OCLC, two newer online services helped propel the growth of IM-based chat reference.

First available in 2006, the web-based Meebo (www.meebo.com) made it much easier to use IM for localized chat reference because library patrons were no longer required to have accounts on a proprietary network, such as AOL or Yahoo, to communicate with librarians. ${ }^{16}$ Instead, Meebo provided web widgets that allowed users to chat via the web browser. Libraries could easily embed these widgets throughout their website and unlike QuestionPoint, Meebo is free and does not require a subscription. Librarians could answer questions using either their account on Meebo's website or by logging-in with a locally installed instant messaging client. In comparison to IM-based chat reference, a number of libraries also found Questionpoint difficult to use due to its complexity and awkward interface. ${ }^{17}$ In 2008, LibraryH3lp (http://libraryh3lp.com) pushed the growth of IM-based chat reference even further because it offered a low-cost, library-specific service that required little technical expertise to implement and operate. LibraryH3lp improved on the Meebo model by adding features such as queues, multi-user accounts, and assessment tools. ${ }^{18}$

IM adds a more informal means of interaction that helps librarians build relationships with their users. Several recent studies have shown that users respond positively to the use of IM for chat reference. The Illinois State University Milner Library found that switching from its older chat reference software to IM increased transactions by 161 percent within one year. ${ }^{19}$ With the introduction of web-based IM widgets Pennsylvania State University Library's IM-based chat reference grew from 20 percent to 60 percent of all virtual reference (VR), which includes email reference, in one year. ${ }^{20}$ A 2010 study of VR and IM service at the University of Guelph Library found 71 percent user satisfaction with IM compared to 70 percent satisfaction with VR overall. ${ }^{21}$ IM use in academic libraries has become ubiquitous, and other types of libraries also use IM to communicate with library patrons.

\section{CASE STUDY}

California State University, San Marcos (CSUSM) is a mid-size public university with approximately 9,500 students. CSUSM is a commuter campus with the majority of students living in North County San Diego and offers many online or distance courses at satellite campuses. The CSUSM Kellogg Library has a robust chat reference service that is used by students on and off campus. The library has about forty-five employees including librarians, library administrators, and library assistants. The following section will discuss the Meebo chat reference pilot, selection of Openfire to replace Meebo, implementation and customization of Openfire, and evaluation of Openfire for chat reference by librarians and as an internal network for all library personnel. 


\section{Meebo Chat Reference Pilot}

To examine the feasibility of using IM for chat reference at CSUSM, the reference librarians initiated a pilot program using Meebo (2008-9). A Meebo widget was placed on the library's homepage, the Ask a Librarian page, and on library research guides. Within the first year of the pilot project, chat reference grew to more than 41 percent of all reference transactions. ${ }^{22}$ Based on responses to user satisfaction surveys, 85 percent indicated they would recommend chat reference to other students, and 69 percent said they preferred it to other forms of reference services. Chat reference is now an integral part of the library's research assistance program, and IM has become a permanent access point for students to contact reference librarians.

Although the new IM service was successful, the pilot program uncovered a number of key shortcomings with Meebo when used for chat reference; these shortcomings are documented in a case study by Meulemans et al. ${ }^{23}$ These findings matched problems reported by other libraries who used Meebo in their reference services. ${ }^{24}$ Meebo is most suited for individual users who communicate one-to-one via IM. For example, Meebo chat widgets are specific to each Meebo user, and it is not possible to share a single widget between multiple librarians. In addition, features such as message queues and message transfers, invaluable for managing a heavily used chat reference service, are not available in Meebo. Those features are essential for working with multiple, simultaneous incoming IM messages, a common occurrence in virtual reference. Other missing features included the lack of built-in transcript retention and lack of automated usage statistics. ${ }^{25}$

\section{Selecting Openfire}

Based on the need for a more robust chat reference system, the CSUSM reference librarians and the web development librarian explored other IM options, especially open-source software. The web development librarian had previous experience using Openfire at the University of Alaska Anchorage, for an internal library IM network and investigated its capabilities to replace Meebo as a chat reference tool.

The desire to replace Meebo for chat reference at CSUSM also provided the opportunity to pilot an internal IM network. Openfire, part of the suite of open-source instant messaging tools from Jive Software, was the only application that could easily fulfill both roles and offered a number of features that made it highly preferable when compared to other IM-based chat reference systems. Of its many features, one of the most valuable was the integration between Openfire user accounts and our campus email system. Being able to tap into the university's email system meant automated configuration and updating of all staff accounts and contact lists. This removed the burden of individual account maintenance associated with external services such as Meebo, LibraryH3lp, and QuestionPoint. Openfire supports internal IM networks at educational institutions such as the University of Pennsylvania, Central Michigan University, and University of California, San Francisco. 
Openfire could meet our IM chat reference needs because it includes the Fastpath plugin, a complete web-based chat management system available at

www.igniterealtime.org/projects/openfire/plugins.jsp. This robust system incorporates important features such as message queues, message transfer, statistics, and canned messages. James Cook University Library in Australia also chose to use Openfire with Fastpath plugin as its chat reference solution based on their need for those features. ${ }^{26}$ Other institutions using Fastpath and Openfire in the role of chat reference or support include the University of Texas, the Oregon/Ohio multistate virtual reference consortium, Mozilla.com, and the University of Wisconsin.

When reviewing chat reference solutions, we considered the possibility of using chat modules available through Drupal (http://drupal.org), the web content management system (CMS) for our library website. The primary advantage of that option was complete integration with the library website and intranet. Further analysis of the Drupal option revealed that the available chat modules where too basic for our needs and that reconfiguration of our intranet and website to incorporate a workable chat reference system would require extensive time. In comparison to the implementation time associated with deploying the Openfire system, using Drupal-based chat modules did not provide a favorable cost-benefit ratio.

While the proprietary LibraryH3lp offered similar functionality for chat reference, its inability to integrate with our email system was clearly a deficit when compared to Openfire. In LibraryH3lp, it is necessary to create accounts for all library personnel in chat reference. Fastpath does not have that requirement if you integrate Openfire with your organization's Lightweight Directory Access Protocol (LDAP) directory. Instead, the system will automatically create accounts for all library staff. Furthermore, the administrative options and interface for Libraryh3lp also did not compare favorably with that of Fastpath. The Fastpath interface for assigning users is more intuitive and the system generates a customizable chat initiation form for each workgroup (figures 1 and 2). Oregon's L-net and Ohio's KnowItNow24x7 offer information about software requirements and an online demonstration of Spark/Fastpath. ${ }^{27}$

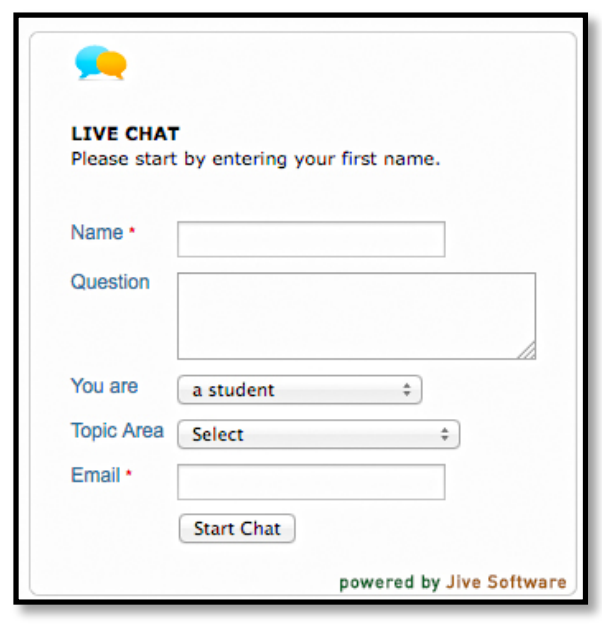


Figure 1. Fastpath Chat Initiation Form for CSUSM Research Help Desk

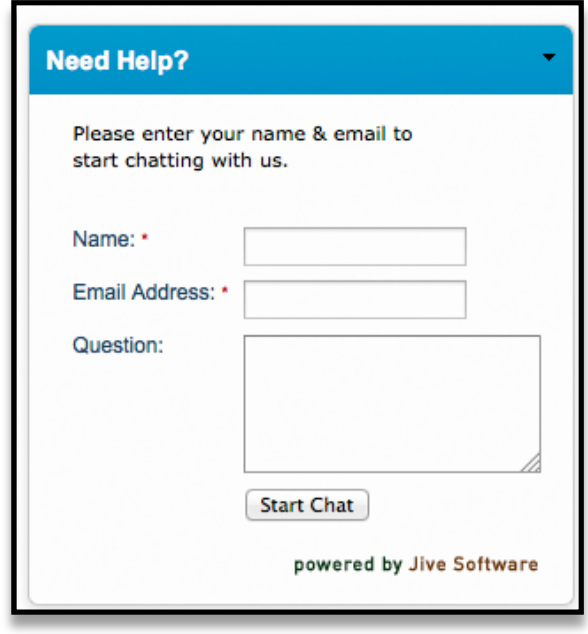

Figure 2. Fastpath Chat Initiation Form for CSUSM Media Library

For our requirements, Openfire was clearly superior to the available systems for chat reference. Its relatively simple deployment requirements and ease of setup helped make it our first choice for building a combined IM network and chat reference system. In the following section, we will discuss the installation, customization, and assessment of our Openfire implementation.

\section{Openfire Installation and Configuration}

The Openfire application is a free download from Ignite Realtime, a community of Jive Software. The program will run on any web server that has a Windows, Linux, or Macintosh operating system. If configured as a self-contained application, Openfire only requires Java to be available on your web server. Installation of the software is an automated process and system configuration is through a web-based setup guide. After the initial language selection form, the next step in the server configuration process is to enter the web server URL and the ports through which the server will communicate with the outside world (figure 3). The third step provides fields for selecting the type of database to use with Openfire and for inputting any information relating to your selection (figure 4).

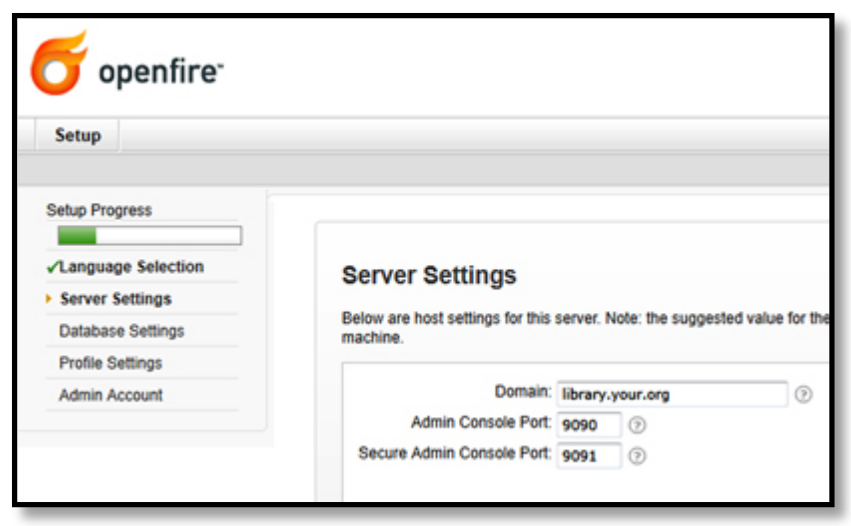


Figure 3. Openfire Server Settings Screen

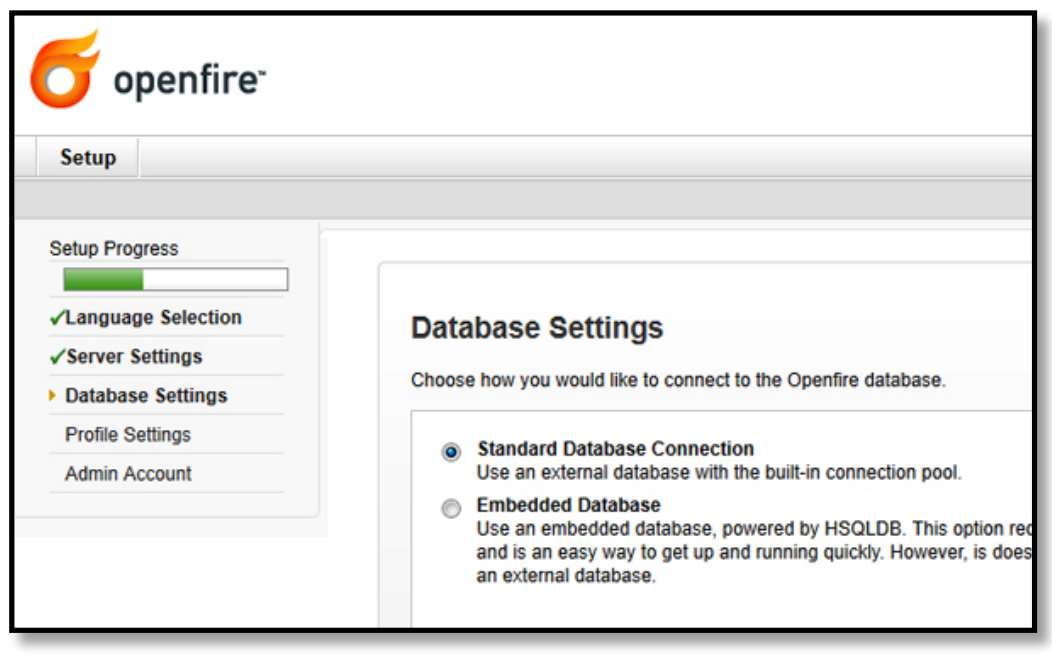

Figure 4. Openfire Database Configuration Form

Openfire uses a database to store information such as IM network settings, user account information, and transcripts. Database options include using an embedded database or connecting to an external database server. Using the embedded database is the simpler option and is helpful if you do not have access to a database server. Connecting to an external database server offers more control of the data generated by Openfire and provides additional backup options. Openfire works with a number of the more commonly used database servers such as MySQL, PostgreSQL, and Microsoft SQL Server. In addition, Oracle and IBM's DB2 are database options with additional free plugins from these vendors. We choose to use MySQL because of our experience using it with other library web applications. If using the external database option, creating and configuring the external database before installing Openfire is highly recommended.

After choosing a database, the Openfire configuration requires the selection of an authentication method for user accounts. One option is to use Openfire's internal authentication system. While the internal system is robust, it requires additional administrative support to manage the process of creating and maintaining user accounts. The recommended option is to connect Openfire with your organization's Lightweight Directory Access Protocol (LDAP) directory (figure 5). LDAP is a protocol that allows external systems to interact with the user information stored in an organization's email system. Using LDAP with Openfire is highly preferable because it simplifies access for your librarians and staff by automatically creating user accounts based on the information in your organization's email system. Library staff simply login with their work email or network account information; they are not required to create a new username and password. 


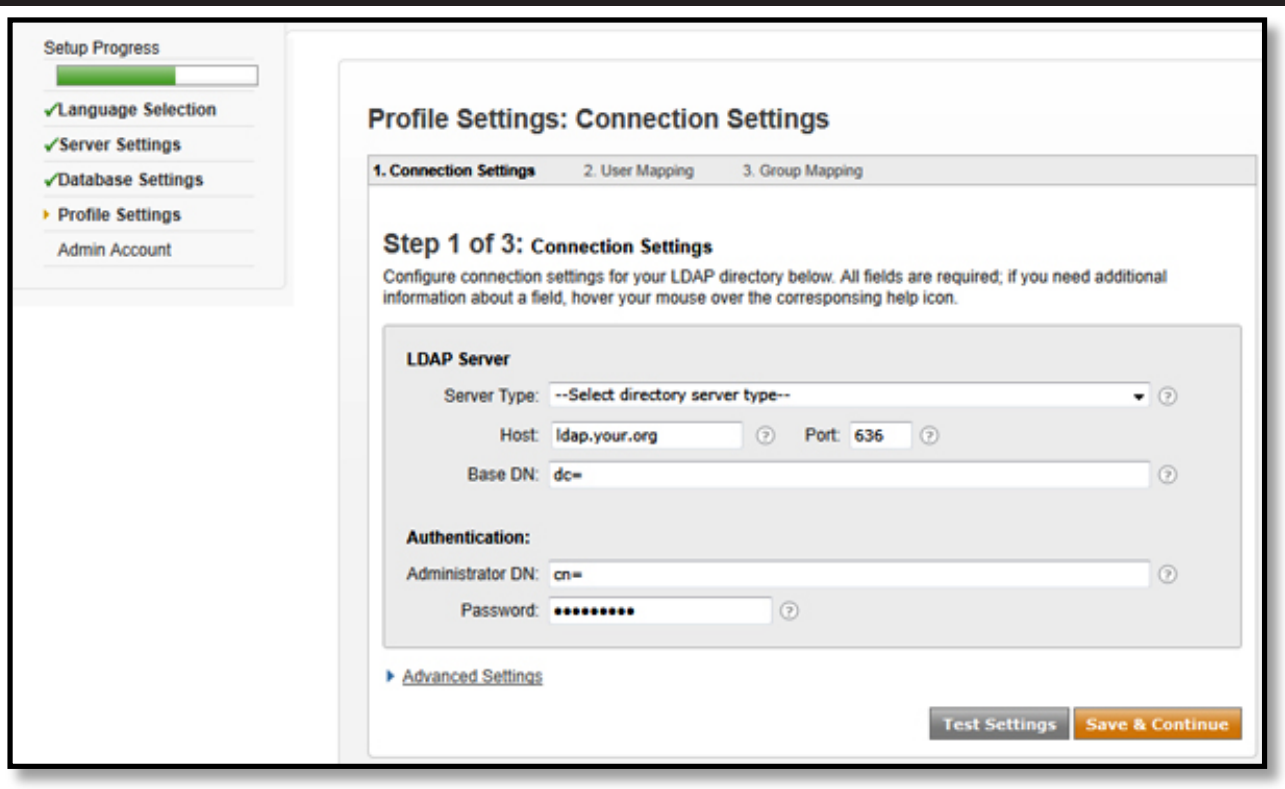

Figure 5. Openfire LDAP Configuration Form

The last step in the configuration process is to grant system administrator access to the appropriate users. If using the LDAP authentication method, you are able to select one or more users in your organization by entering their email id (the portion before the ampersand). The selected users will have complete access to all aspects of the Openfire server.

Once the setup and configuration process is complete, the server is ready to accept IM connections and route messages. Reviewing the settings and options within the Openfire system administration area is highly recommended. Most libraries will likely want to adjust the configurations within the sections for server settings and archives.

\section{Connecting the IM Network}

The second phase of the implementation process connected our Library personnel with the IM network using IM software installed on their workstations. The Openfire IM server works with any multiprotocol IM client ("multiprotocol" refers to support for simultaneous connections to multiple IM networks) that provides options for configuring an XMPP or Jabber account. Some of the more popular IM clients that offer this functionality include Spark, Trillian, Miranda, and Pidgin.

Based on our chat reference requirements, we choose to use Spark (www.igniterealtime.org/projects/spark), an IM client program designed to work specifically with the Fastpath web chat service. Spark comes with a Fastpath plugin that enables users to receive and send messages to anyone communicating through the web-based Fastpath chat widgets (more information on Fastpath configuration is in the next section of this article). This plugin provides a tab for logging into a Fastpath group and for viewing the status of the group's message queues 
(figure 6). Spark also includes many of the features offered by other IM clients including built-in screen capture, message transfer, and group chat.

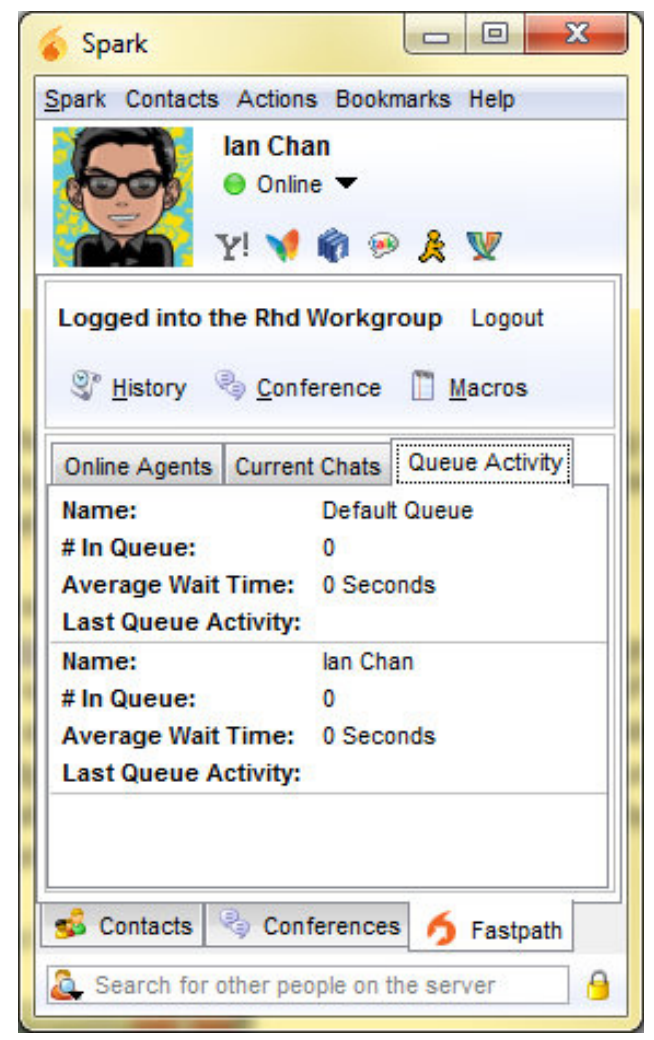

Figure 6. The Fastpath Plugin for Spark

Library personnel were able to install Spark on their own by downloading it from the Ignite Software website and launching the software's installation package. The installation process is very simple and user-specific information is only required when Spark is started for the first time. The fields required for login include the username and password of the user's organizational email and the address of the IM server. As part of our implementation process, we also provided library staff with recommendations regarding the selection and configuration of optional settings that might enhance their IM experience. Recommendations included auto-start of Spark when loggingin to computer and the activation of incoming message signals, such as sound effects and pop-ups.

On our Openfire server, we had also installed the Kraken Gateway (http://kraken.blathersource.org) plugin to enable connections to external IM networks. The gateway plugin works with Spark to integrate library staff accounts on chat network such as Google Talk, Facebook, and MSN (an example of integrated networks is shown in figure 6.) By integrating Meebo as well, librarians were able to continue using the Meebo widgets they had embedded into their research guides and faculty profile pages. This allowed them to use Spark to receive IM messages rather than logging on to the Meebo website. 


\section{Configuring the Fastpath Plugin for Chat Reference}

A primary motivation for using Openfire was the feature set available in the Fastpath plugin. Fastpath is a complete chat messaging system that includes workgroups, queues, chat widgets, and reporting. Fastpath actually consists of two plugins that work together, Fastpath Service for managing the chat system and Fastpath Webchat for web-based chat widgets. Both plugins are available as free downloads from the Openfire Plugins section of the Ignite Software websitewww.igniterealtime.org/projects/openfire/plugins.jsp.

To install Fastpath, upload the its packages using the form in the plugins section of the Openfire administrative interface. The plugins will automatically install and add a Fastpath tab to the administrative main menu. The first step in getting started with the system is to create a workgroup and add members (figure 7). Within each new workgroup, one or more queues are required to process and route incoming requests and each queue requires at least one "agent." In Fastpath, the term agent refers to those who will receive the incoming chat requests.

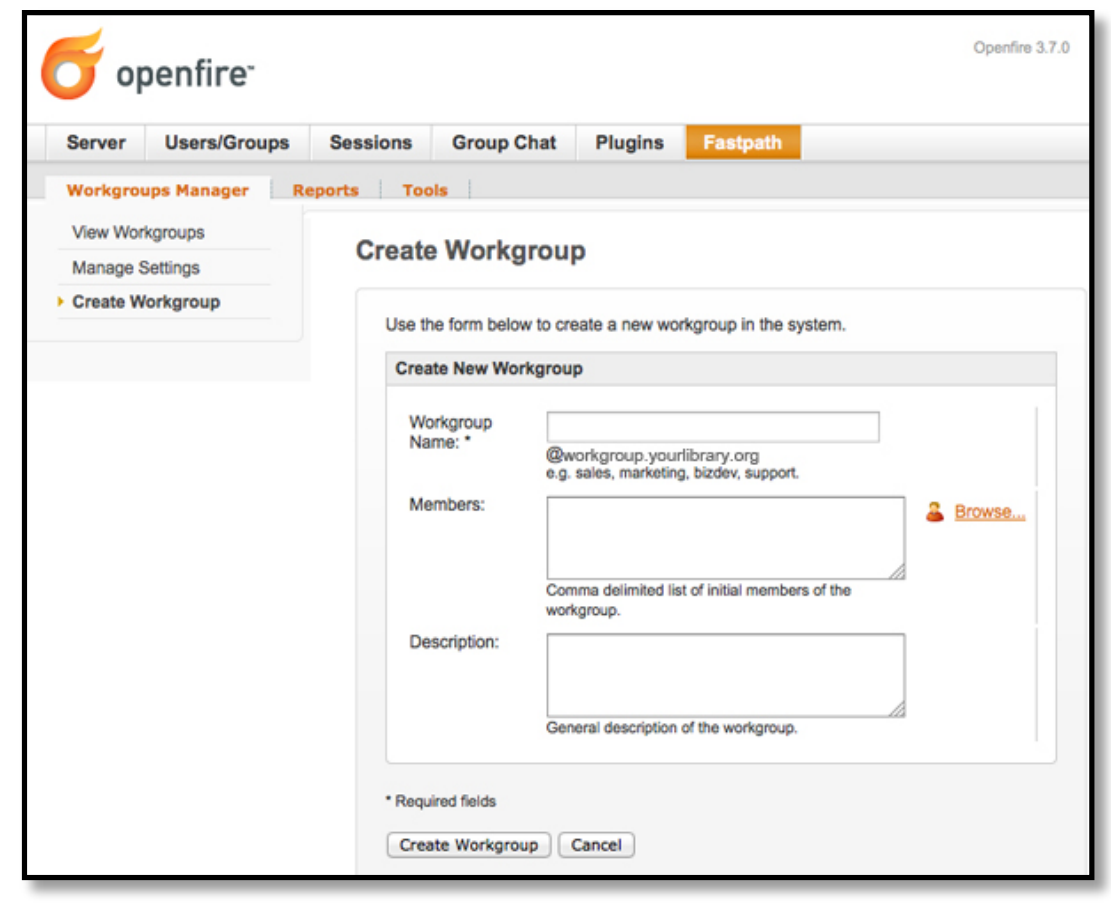

Figure 7. Workgroup Setup Form in Fastpath

As work groups are created, the system automatically generates a chat initiation form which by default includes fields for name, email and question. Administrators can remove, modify, and add any combination of field types including text fields, dropdown menus, multiline text areas, radio buttons, and check boxes. You may also configure the chat initiation form to require completion of some, all, or none of the fields. At CSUSM, our form (figures 1 and 2) includes name, question, email, and a dropdown menu for selecting the topic area of the user's research and a field for the user to enter their question. The information in these fields allows us to quickly route incoming 
questions to the appropriate subject librarian. Fastpath includes the ability to create routing rules that use the values submitted in the form to send messages to specific queues within a workgroup. In future, we may use the dropdown menu to automatically route questions to the subject specialist based on the student's topic.

There are two methods to make the Fastpath chat widget available to the public. The standard approach embeds a presence icon on your webpage and provides automatic status updates. Clicking on the icon displays the chat initiation form. For our needs we choose to embed the chat initiation form in our webpages (see appendix B for sample code). When the user submits the form, Openfire routes the message to the next available librarian. On the librarian's computer, the Spark program plays a notification sound and displays a pop-up dialog. The pop-up dialog remains open until the librarian accepts the message, passes it on, or the time limit for acceptance is reached, in which case the message returns to the queue for the next available librarian.

\section{Evaluation of Openfire for Enhanced Chat Reference}

The CSUSM reference librarians found Fastpath and Openfire to be much more robust than Meebo for chat reference. The ability to keep chat transcripts and to retain metadata such as time stamps, duration of chats, and topic of research for each conversation is very helpful toward analyzing the effectiveness of chat research assistance and for statistical reporting. The automated recording of transcripts and metadata saved time when compared to Meebo. Using Meebo, transcripts were manually copied into a Microsoft Word document and the tracking statistics of IM interactions were kept in a shared Excel spreadsheet. Other useful features of Fastpath were the capability of transferring of patrons to other librarians and having more than one librarian monitor incoming questions. Furthermore, access to the database holding the Fastpath data allowed us to build an intranet page to monitor real-time incoming IM messages and their responses.

However, some issues were encountered with the Fastpath plugin when initiating chat connections. We experienced intermittent, random instances of dropped IM connections and lost messages. While many of these lost connections were likely the result of user actions (accidentally closing the chat pop-up, walking away from the computer, etc.), others appear to have been due to problematic connections between the server and the user's browser. To address these issues, we are now asking users to provide their email when they initiate a chat session. With user emails and our real-time chat monitoring system, we are able to follow up with reference patrons that experience IM connection issues and provide research assistance via email.

\section{Evaluation of Openfire as an Internal Communication Tool}

While the adoption of IM as internal communication tool was highly encouraged, its use was not mandatory for all library personnel. Based on the varied technical background of our staff and librarians, we recognized that some might find IM difficult to integrate within their workflow or communication style and chose a soft-launch for our network. 
In summer 2011, we conducted a survey of CSUSM library personnel (44 respondents, 99 percent of total staff) to evaluate IM as an internal communication tool. (See appendix A for survey questions.) We found that 59 percent of staff use the internal IM network while 85 percent use some type of IM for web-based chat for work. Of those who use internal IM, 30 percent used it daily. While the survey was anonymous, anecdotal discussions indicate adoption rates are higher among library units where the work is technically oriented or instructional in nature, such as Library Systems and the Information Literacy Program/Reference. Among the respondents who use IM, 45 percent of library staff indicated they use it because it allows quick communication between those in the library and 39 percent like its informal nature of communication. Twenty percent of total respondents preferred IM to email and phone communications. Two respondents use the internal IM network but were dissatisfied with it and indicated it did not work well while one found it too difficult to use.

An additional survey question was geared for staff members who do not use the internal IM network at all ("Why do you not use the Library IM network?"). This question was designed to find areas of possible improvement within our system to encourage greater use. Survey respondents were allowed to select more than one reason. The most common reasons given by those who do not use the library IM network were that they don't feel the need (34 percent of nonusers), they mainly communicate with staff members who are also not utilizing the IM network (18 percent), IM does not work for their communication style (14 percent), and privacy concerns (14 percent). We believe more in-depth analysis is necessary to learn more regarding the perceived usefulness of IM within our organization and to further its adoption.

\section{CONCLUSION}

Through additional training and user education, we hope to promote greater use of the Openfire internal IM network among those who work in the library. While 100 percent adoption of IM as a communication tool is not a stated goal of our project, we believe that some staff have not realized the full potential of IM for collaboration and productivity due to a lack of experience with this technology. In hindsight, additional training sessions beyond the initial introductory workshop to set up the Spark IM client may have increased the usage of IM by staff. For example, providing more information on the library's policies regarding internal IM tracking and the configuration of our system may have alleviated concerns regarding privacy. In addition, we need to lead more discussions on the benefits of IM for collaboration, lowering disruptions, and increasing effectiveness in the workplace.

Openfire and Fastpath for chat reference has brought many new features that were previously unavailable to chat reference at CSUSM. The addition of queues, message transfer, and transcripts has enhanced the effectiveness of this service and eased its management. Compared to the prior chat reference implementations that used QuestionPoint and Meebo, this new system is more user friendly and robust. 
Furthermore, the internal IM network and its connection to web-based chat widgets offer the opportunity for building a library that is more open to users. Library users could feasibly contact any library staff member, not just reference librarians, via IM for help. We are testing this concept with a pilot project involving the CSUSM Media Library. They are staffing their own chat workgroup and a chat widget is now available on their website. In the future, we also hope to employ a chat widget for Circulation and ILL services, another public services area that frequently works with library users.

It is important to note that the success of Openfire and IM in the library attracted the attention of other CSUSM instructional and student support areas. In spring 2011, Instructional and Information Technology Services (IITS), which provides campus-wide technology services for faculty, staff, and students piloted an Openfire-based IM helpdesk service to assist users with technology questions and problems. As of fall 2011, the "Ask an IT Technician" service is fully implemented and available on all campus webpages. Discussions on the adoption of IM for other campus student services, such as financial aid and counseling, have also occurred. In addition to being a contact point for students, IM has potential to improve the internal communication within the organization.

\section{REFERENCES}

1. Hee-Kyung Cho, Matthias Trier, and Eunhee Kim, "The Use of Instant Messaging in Working Relationship Development: A Case Study," Journal of Computer-Mediated Communication 10, no. 4 (2005), http://onlinelibrary.wiley.com/doi/10.1111/j.1083-6101.2005.tb00280.x/full (accessed Aug. 1, 2011).

2. Bonnie A. Nardi, Steven Whittaker, and Erin Bradner, "Interaction and Outeraction: Instant Messaging in Action," in Proceedings of the 2000 ACM Conference on Computer Supported Cooperative Work (New York, New York: ACM Press, 2000),79-88.

3. Ellen Isaacs et al., "The Character, Functions, and Styles of Instant Messaging in the Workplace," in Proceedings of the 2002 ACM Conference on Computer Supported Cooperative Work (New York, New York: ACM Press, 2002), 11-20.

4. Victor M. González and Gloria Mark, "Constant, Constant, Multi-tasking Craziness: Managing multiple working spheres," in Proceedings of the SIGCHI Conference on Human Factors in Computing Systems (New York, New York: ACM Press, 2004), 113-20.

5. R. Kelly Garrett and James N. Danziger, "IM = Interruption Management? Instant Messaging and Disruption in the Workplace," Journal of Computer-Mediated Communication 13, no. 1 (2007), http://jcmc.indiana.edu/vol13/issue1/garrett.html (accessed Jun. 15, 2011).

6. Nardi, Whittaker, and Bradner, "Interaction and Outeraction," 83.

7. Albert H. Huang, Shin-Yuan Hung, and David C. Yen, "An Exploratory Investigation of Two Internet-based Communication Modes," Computer Standards \& Interfaces 29, no. 2 (2006): 238-43. 
8. Anabel Quan-Haase, Joseph Cothrel, and Barry Wellman, "Instant Messaging for Collaboration: A Case Study of a High-Tech Firm," Journal of Computer-Mediated Communication 10, no. 4 (2005), http://jcmc.indiana.edu/vol10/issue4/quan-haase.html (accessed Jun. 12, 2011).

9. Carol X. J. Ou et al., "Empowering Employees through Instant Messaging," Information Technology \& People 23, no. 2 (2010): 193-211.

10. Cho, Trier, and Kim, "Instant Messaging in Working Relationship Development."

11. Lynn Wu et al., "Value of Social Network-A Large-Scale Analysis on Network Structure Impact to Financial Revenue of Information Technology Consultants" (paper presented at Winter Information Systems Conference, Salt Lake City, UT, Feb. 5, 2009).

12. Pruthikrai Mahatanankoon, "28P. Exploring the Impact of Instant Messaging on Job Satisfaction and Creativity," CONF-IRM 2010 Proceedings (2010).

13. Ashish Gupta and Han Li, "Understanding the Impact of Instant Messaging (IM) on Subjective Task Complexity and User Satisfaction," in PACIS 2009 Proceedings. Paper 10, http://aisel.aisnet.org/pacis2009/1; and Stephanie L. Woerner, JoAnne Yates, and Wanda J. Orlikowski, "Conversational Coherence in Instant Messaging and Getting Work Done," in Proceedings of the 40th Annual Hawaii International Conference on System Sciences, http://www.computer.org/portal/web/csdl/doi/10.1109/HICSS.2007.152 (2007).

14. Marshall Breeding, "Instant Messaging: It's Not Just for Kids Anymore," Computers in Libraries 23, no. 10 (2003): 38-40.

15. John Fink, “Using a Local Chat Server In Your Library," Feliciter 56, no. 5 (2010): 202-3.

16. William Breitbach, Matthew Mallard, and Robert Sage, “Using Meebo's Embedded IM for Academic Reference Services: A Case Study," Reference Services Review 37, no. 1 (2009): 83-98.

17. Cathy Carpenter and Crystal Renfro, "Twelve Years of Online Reference Services at Georgia Tech: Where We Have Been and Where We Are Going," Georgia Library Quarterly 44, no. 2 (2007), http://digitalcommons.kennesaw.edu/glq/vol44/iss2/3 (accessed Aug. 25, 2011); and Danielle Theiss-White et al., "IM'ing Overload: Libraryh3lp to the Rescue," Library Hi Tech News 26, no. 1/2 (2009): 12-17.

18. Theiss-White et al., "IM'ing overload," 12-17.

19. Sharon Naylor, "Why Isn't Our Chat Reference Used More?" Reference \& User Services Quarterly 47, no. 4 (2008): 342-54

20. Sam Stormont, "Becoming Embedded: Incorporating Instant Messaging and the Ongoing Evolution of a Virtual Reference Service," Public Services Quarterly 6, no. 4 (2010): 343-59. 
21. Lorna Rourke and Pascal Lupien, "Learning from Chatting: How our Virtual Reference Questions are Giving Us Answers," Evidence Based Library \& Information Practice 5, no. 2 (2010): 63-74.

22. Pearl Ly and Allison Carr, "Do u IM?: Using Evidence to Inform Decisions about Instant Messaging in Library Reference Services" (poster presented at the 5th Evidence Based Library and Information Practice Conference, Stockholm, Sweden, June 29, 2009), http://blogs.kib.ki.se/eblip5/posters/ly carr poster.pdf (accessed August 1, 2011).

23. Yvonne Nalani Meulemans, Allison Carr, and Pearl Ly, "From a Distance: Robust Reference Service via Instant Messaging," Journal of Library \& Information Services in Distance Learning 4, no. 1 (2010): 3-17.

24. Theiss-White et al., "IM'ing overload," 12-17.

25. Meulemans, Carr, and Ly, "From a Distance," 14-15

26. Nicole Johnston, "Improving the Reference and Information Experience of Students in Regional Areas-Does an Instant Messaging Service Make a Difference?" (paper presented at 4th ALIA New Librarians Symposium, December 5-6, 2008, Melbourne, Australia), http://eprints.jcu.edu.au/2076(accessed August 17, 2011); and Alan Cockerill, "Open Source for IM Reference: OpenFire, Fastpath and Spark" (workshop presented at Fair Shake of the Open Source Bottle, Griffith University, Queensland College of Art, Brisbane, Australia, November 20, 2009), http://www.quloc.org.au/download.php?doc id=6932\&site id=255 (accessed August 4, 2011).

27. Oregon State Multistate Collaboration, "Multi-State Collaboration: Home," http://www.oregonlibraries.net/multi-state (accessed August 16, 2011). 
APPENDIX A

\section{Library Instant Messaging (IM) Usage Survey}

The information you submit is confidential. Your name and campus ID are NOT included with your response.

Which of the following do you use...

\begin{tabular}{|l|l|l|}
\hline & for work & $\begin{array}{l}\text { for } \\
\text { personal }\end{array}$ \\
\hline Library's IM Network (Spark) & & \\
\hline Meebo & & \\
\hline MSN & & \\
\hline Yahoo & & \\
\hline GTalk & & \\
\hline Facebook or other website-specific chat system & & \\
\hline IM app on my phone & & \\
\hline Trillian, Pidgin or other IM aggregator & & \\
\hline Skype & & \\
\hline Other & & \\
\hline
\end{tabular}

If you selected other, please describe: 
On average, how often do you communicate via IM or web-based chat at work?

- Several times a day

- Almost daily

- Several times a week

- Several times a month

- Never

How often do you use IM or web-based chat to ...

\begin{tabular}{|c|c|c|c|c|c|}
\hline & 5-Often & 4 & $\begin{array}{l}3- \\
\text { Sometimes }\end{array}$ & 2 & 1 -Never \\
\hline discus & & & & & \\
\hline sociali & & & & & \\
\hline $\begin{array}{l}\text { answe } \\
\text { library }\end{array}$ & & & & & \\
\hline $\begin{array}{l}\text { talk ab } \\
\text { relatec }\end{array}$ & & & & & \\
\hline reques & & & & & \\
\hline Other & & & & & \\
\hline
\end{tabular}

If you selected other, please describe:

If you use IM to communicate at work, what do you like about it?

- Allows for quick communication with others in the library

- Facilitates informal conversation

- Students like to use it to ask library related questions

- I prefer IM over phone or email

- Other: 
Why do you NOT use the Library IM network?

- Don't feel the need

- The people I usually talk to aren't on it

- Does not work well

- Never get around to it . . . but would like to

- It doesn't work for my communication style

- The system is too difficult to use

- Privacy concerns

- Other:

Additional comments? 


\section{APPENDIX B}

\section{IFRAME Code for Embedding Fastpath Chat Widget}

<iframe scrolling= "no" frameborder= "0" src="http://library.your_org.edu:

7070/webchat/userinfo.jsp?workgroup=<workgroupname>@workgroup.library.your_org.edu”>

Your browser does not support inline frames or is currently configured not to display inline frames. Content can be viewed at actual source page: http://library.your_org.edu:

7070/webchat/userinfo.jsp?workgroup=<workgroupname>@workgroup.library.your_org.edu $</$ iframe $>$ 